\title{
Application of Improved Expert Scoring Method with Delphi Principle in CMMI DAR Process Area
}

\author{
Junwei $\mathrm{Hou}^{1, a}$, Xianggu Meng ${ }^{2, \mathrm{~b}}$ \\ ${ }^{1,2}$ CASIC Intelligence Industry Development Co. Ltd, No 50.Yongding Road, Haidian Distract, Beijing, \\ China \\ ahoujunwei8703@126.com, ${ }^{b}$ meng6233@sina.com
}

\section{Keywords: CMMI DAR Process Area Expert Scoring Delphi Smart City}

\begin{abstract}
CMMI (Capability Maturity Model Integration) is the key architecture of software engineering management which divides the whole software development life cycle into twenty-two process areas. DAR (Decision Analysis and Resolution) process area is one of the key process areas, it is executed when we meet multiple candidate programs. The main problem of the conventional expert scoring method, which is the most popular method for DAR, is difficult to converge to the best project objectively. In this paper, the Delphi principle was proposed to improve the expert scoring method in the DAR process area. And a smart city project was evaluated at project setting stage to show the improved method is effective and objective.
\end{abstract}

\section{Introduction}

With the rapid development of information technology, software development process is even more important. In the area of software engineer management, largely models are emerging, and CMMI is one of them. CMMI (Capability Maturity Model Integrated), is a software development framework which is researched by the United States Department of Defense. The purpose of the method is to help software companies to manage and improve the software engineering process, so as to enhance development capabilities and improve software quality [1]. CMMI contains twenty-two process areas, composed of four parts: organizing, project management, engineering, and supporting. DAR (Decision Analysis and Resolution) is a process area of supporting part through the whole software development circle. The twenty-two process areas of CMMI are as follows (Fig. 1):

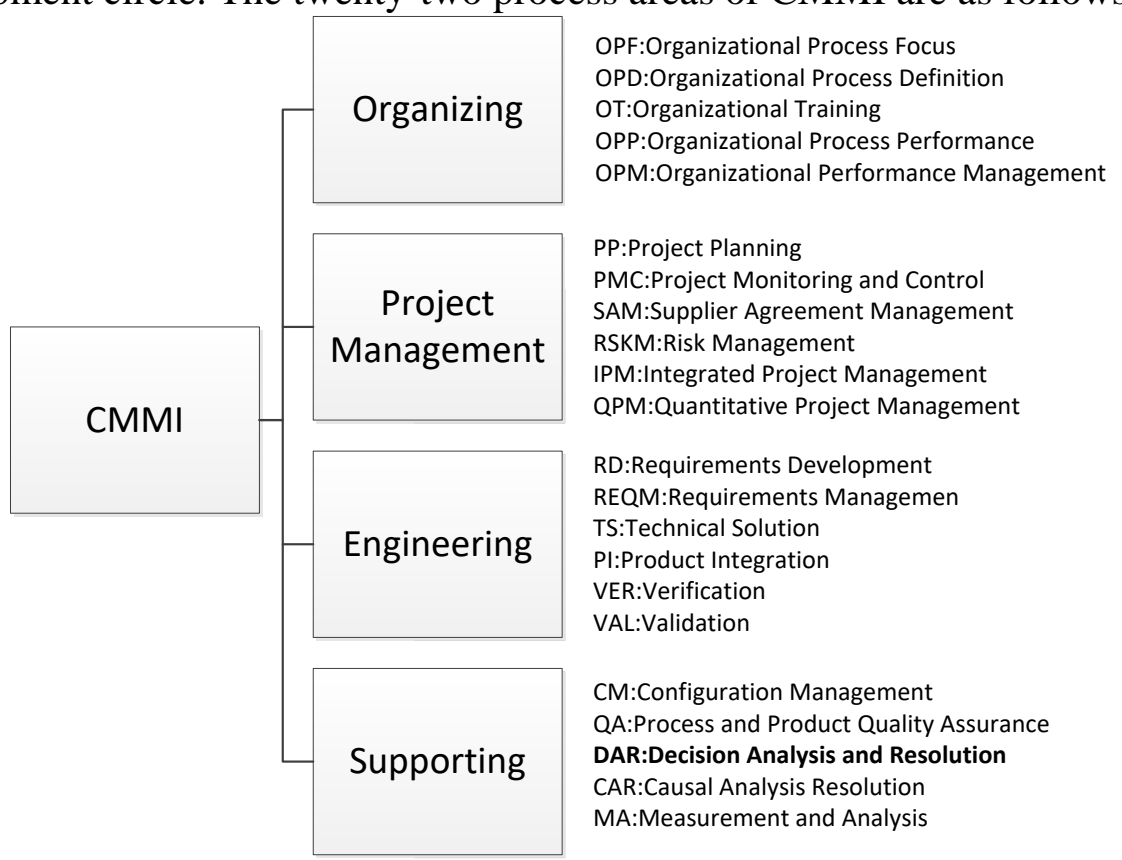

Fig. 1 Process Areas of CMMI

DAR, which means that, we formulate an evaluation process, evaluate candidates based on specific criteria, and make decisions which candidate is best. It is carried out when we face more than 
twice options, like solutions, design architectures, developing tools and so on. [2] It can be used in project setting stage, risk management, design stage and so on. Expert scoring method is one of the majority DAR methods. In this paper, we introduce Delphi expert scoring method, which is based on the Delphi theory and experts scoring method. Using this improved strategy in DAR process, we get the optimal choice.

\section{DAR Process Area of CMMI}

The purpose of DAR is analyzing possible decisions using a formal evaluation process that evaluates identified alternatives against established criteria. The steps of DAR are as follows (Fig. 2):

First, select the decision point (some issues needs DAR process ), such as a variety of projects selection, design options, risk response measures, multi-vendor selection, a variety of development tools, etc.

Second, establish the evaluation criteria, according to that decided, different selections with different evaluation criteria, such as the project setting, you can consider the demand certainty, advanced technology, realizable, market prospects, economic analysis, etc.

Third, choice evaluation methods, such as prototype simulation, brainstorming, decision tree analysis, earned value analysis, expert scoring, etc.

Fourth, evaluate alternatives.

Fifth, choice the best one.

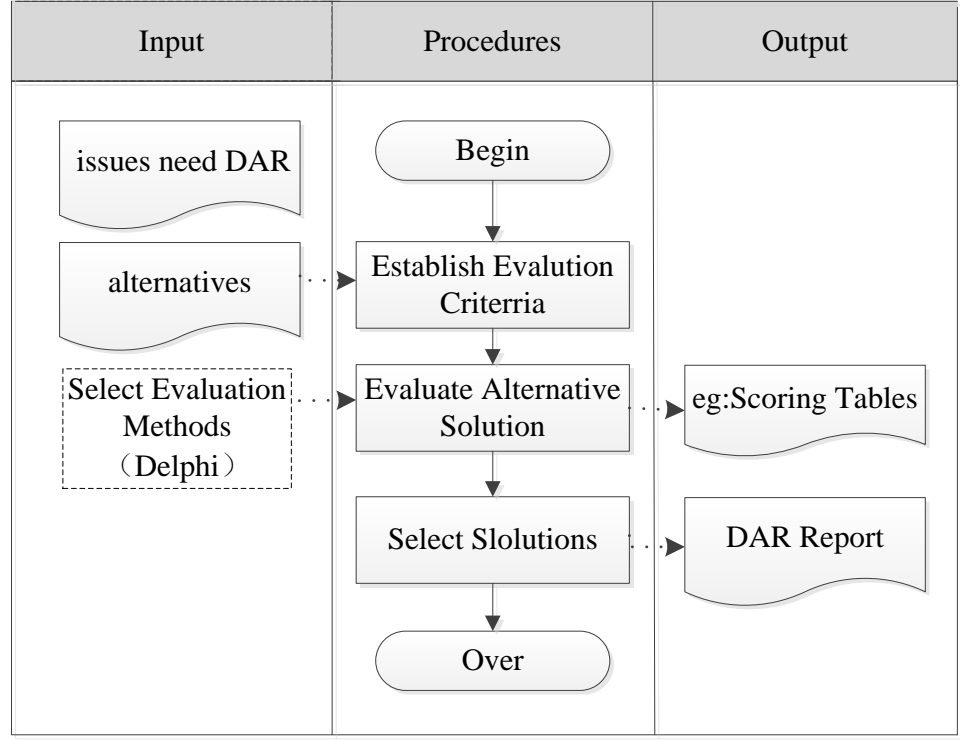

Fig. 2 DAR Steps

The methods we used to evaluate alternatives is very important, and we research a new method, that is Delphi expert scoring method.

\section{Delphi expert scoring method}

The expert scoring method, which means that we establish evaluation criteria, determine the weights of each criteria, and then ask some representative experts score alternative schemes according to the evaluation criteria, then collect the scoring tables, decide the best selection with highest score. Expert scoring method using the weighted method to analysis and score alternatives, an expert on the total score of the $\mathrm{j}$-th program:

$$
W_{j}=\sum_{i=}^{n} A_{i} W_{i}
$$

In this formula, $\mathrm{Wj}$ is an expert evaluation of the $\mathrm{j}$-th scheme, $\mathrm{Wi}$ is the score of the $\mathrm{i}$-th criteria 
of the $\mathrm{j}$-th program, and $\mathrm{Ai}$ is the weight of the $\mathrm{i}$-th criteria. The average score of the $\mathrm{j}$-th program is the average of the scores of all experts. Finally, according to the average of each scheme, the best solution is obtained.

Usually, we use expert scoring method through expert committee review meeting, experts sit together to discussing, grading, and selects the optimal plan. But in this way, may be some expert's insights twist after discussion, or some expert has biased ideas so greatly affect the average. In order to solve the problem, we find a more objective way to avoid subjective conclusion due to bias.

Delphi method (as known as experts inquiry method), is a method of feedback through anonymous survey. Delphi's principle is back to back, so that each expert make their own judgement independently and freely, the advantage of this method is to fully absorb the expert opinions, reduce the unreasonable differences due to prejudice or arbitrary [3]. The steps are as follows: first, discuss the questions to the experts, ask for ideas, and gather comments, then summary and analysis. According to the problems of large deviation, we give advice and feedback to experts, and inquiry again. Repeated several rounds, the views of experts gradually consistent at last.

We combine Delphi and expert scoring method, when inquiring, the score tables sent to the assessment experts, each expert according to the choice of program, scored and feedback. After receiving the feedback forms, if there are some extremely deviation at some factors, we will give the analysis to the experts, and collect feedbacks again. After repeated several times, that approaching the coincident scoring results, the highest scoring scheme as the optimal scheme.

\section{The Application of Delphi Expert Scoring Method in the Decision of Smart Cities Research Project}

Smart city which is using information and communication technology, sense, analyze and integrate all the key information of the core system of urban operation, so as to make intelligent response of various needs including the people's livelihood, environmental protection, public security, city services, industrial and commercial activities [4-5]. Smart city solution contains big data, network+, cloud computing and other mainstream advanced technologies. Here, we use the CMMI DAR process in the choice of research and development (R\&D) projects, with the improvement Delphi expert scoring method. First of all, solicit the views of engineers, draw several alternative programs; secondly, determine the evaluation criteria, in four aspects as consideration:

Market prospect: including market space (MS), competitive ability (CA), marketing plan (MP);

Technical indicators: including technical innovation (TI), demand analysis (DA), product features (PF), the scope of the business boundary definition (SBBD);

Organization and Implementation: project team organization rationality (PTOR), progress management (PM);

Input and output: three year economic benefit (EB), input output ratio (IOR).

According to these factors, each factor defines the weight of score. Three projects' scoring evaluation tables sent to 4 experts, scoring. The first round of collecting feedback results, make analysis, summed up the deviation of the factors, and feedback to the experts. Experts will make adjustments based on recommendations, feedback again. After the 3 rounds, the score reach consistent results, the project selected according to the highest total score. The 3 rounds of the survey is as follows (Fig.3-5).

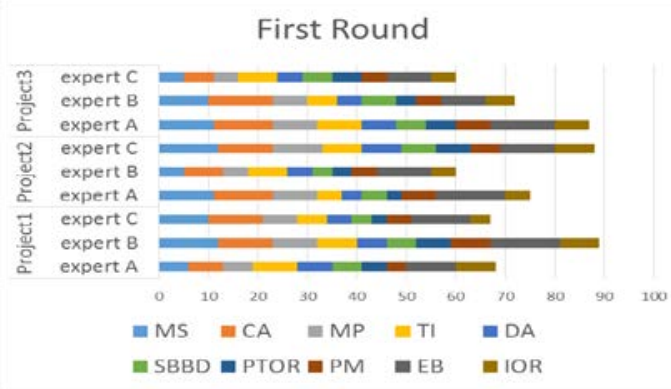

Fig. 3 First Round Expert Scoring (Conventional)

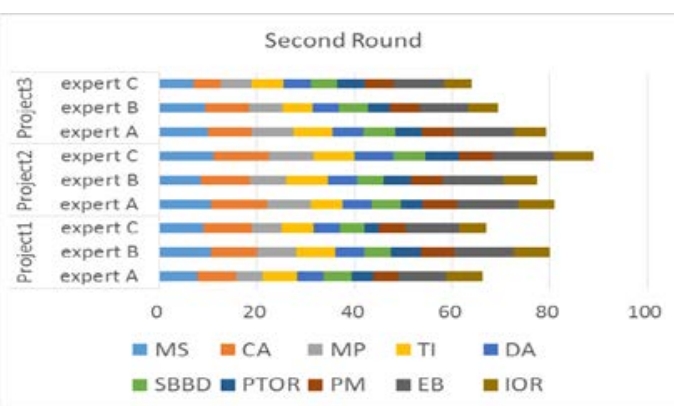

Fig. 4 Second Round Expert Scoring 


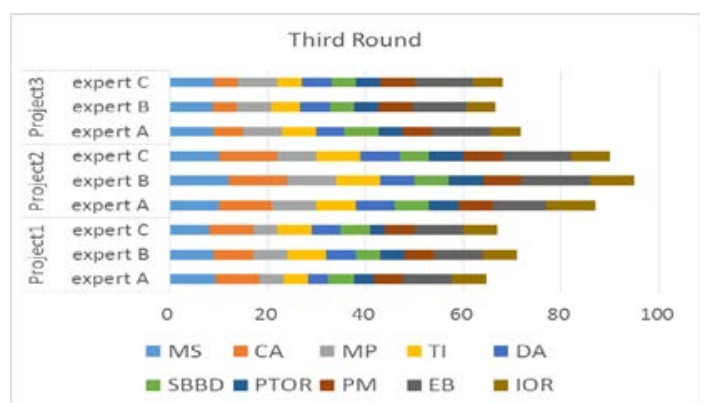

Fig. 5 Third Round Expert Scoring

For the analysis chart shown above, we can know, in the first round, experts obtained different scores and hold on the optimal solution diffrently according to their own opinions.With analysis and advice, after the second round results feedback, the scoring of various factors became less diffirent. In the third round, the experts are more consistent, unified the highest scoring project 2 as the R\&D project decision. Therefore, through the Delphi expert scoring method, the decision of the project is the most feasible.

\section{Conclusion and Prospect}

With the rapid growth of information technology, the software industry has been developed both in management level and technical level for nearly a century. CMMI has been widely used by many software companies to evaluate the capability of software management level, and is usually referenced and highly approval. Meanwhile the twenty-two process areas of CMMI are more and more mature and improved in constant. DAR is the necessary process in software development. The practice shows that the application of the improved Delphi expert scoring method can improve the effectiveness, feasibility and specialization in DAR domain. In the near future, we will study to applying this method to risk management, design scheme selection and implementation plan selection, etc.

\section{References}

[1] Huibin Shang, Analysis and comparison of CMMI and agile development process, Telecom World, No.13 (2016)

[2] Jun Xu, Yufeng Lu, Jianxi Peng, Research the implementation method of DAR process in CMMI model, Software Guide, Vol.9 No.11 (2010).

[3] Xiaoliang Hou: CMMI-BASED Software Project Risk Management -A Case of ABC on the Development of CCIF. (2012)

[4] Jingyuan Wang, Chao Li, Zhang Xiong, Zhiguang Shan, Summary of smart city research based on Data, Joural of Computer Research and Development,51(2):p.239-259(2014)

[5] COOPER Dave E, Intelligent transportation systems for smart cities: a progress review, Vol.12 (2012). 